\title{
Rhenium Effect in Irradiated Mo-Re Alloys and Welds
}

\author{
Alexander Krajnikov ${ }^{1, *}$, Fumio Morito ${ }^{2}$, Mykola Danylenko ${ }^{1}$ \\ ${ }^{1}$ Frantsevich Institute for Problems of Materials Science, Krzhizhanovsky St. 3, Kyiv, 03680, Ukraine \\ ${ }^{2}$ MSF Laboratory, 4-20-12, Keyakidai, Moriya, 302-0128, Japan \\ *Corresponding Author: avk@ipms.kiev.ua
}

Copyright (C) 2014 Horizon Research Publishing All rights reserved.

\begin{abstract}
The mechanical properties and microstructure of Mo-Re alloys and welds were examined after low- and high-temperature irradiation focusing on the effects of Re concentration, phase stability, microstructural changes and impurity redistribution. Ductility improvement and fracture mode changes from intergranular to transgranular are clearly observed in Mo-Re alloys with an increase in Re content. Neutron irradiation causes hardening of the matrix and concurrently reduces its ductility. The hardening effect is rather limited and unstable after low-temperature irradiation because of the lack of ductility. The damaging effect of neutrons is smaller and hardening of the matrix is more pronounced after high-temperature irradiation. Intensification of homogeneous nucleation of Re-rich phases throughout the bulk is observed after high-temperature irradiation in all of the Mo-Re alloys studied. As a result, the microstructure and strength of all parts of as-irradiated welds equalize and show approximately the same level of strength.
\end{abstract}

Keywords Mo-Re Welds, Re-Effect, Tensile Properties, Neutron Irradiation, Radiation-Induced Hardening, Phase Transformations

\section{Introduction}

The beneficial effect of rhenium alloying at both low-and high-temperatures and the mechanical properties of Mo-Re alloys has been known since the late 1950s [1,2]. Several Mo-Re alloy compositions have been developed and used commercially in various applications. Nevertheless, the development of new technologies offers new challenges in the field of Mo-Re materials every year. For example, nowadays, structural materials for fusion reactors or space nuclear power systems are subject to constant discussion $[3,4]$. Resistance to neutron irradiation, weldability and phase stability are the key factors for the successful application of Mo-Re alloys in the given field.

Low-alloyed Mo and Mo-Re alloys are known to be very susceptible to manufacturing technologies and heat treatment regimes. Control of interstitial impurities with the aim of reducing the oxygen content and proper welding regimes allow one to produce sound Mo-Re welds in electron beam welding. Intergranular embrittlement of weld metal can be improved by pre-weld and/or post-weld heat treatments, which are effective at recovering the alloy strength and plasticity [5-7].

Neutron irradiation causes strong hardening of the matrix and simultaneously reduces the plasticity of Mo-based alloys. Dependence of the radiation effects on the metallurgical factors, such as fabrication process, alloy composition, heat treatment, irradiation conditions, has been intensively studied in both low-alloy Mo and Mo-Re alloys [8-15]. Despite evident progress being achieved in the understanding of radiation-induced embrittlement, prospects for practical usage of Mo base alloys in fusion reactor elements are, however, still under discussion.

The paper focuses on the study of tensile properties and microhardness of Mo-Re welds irradiated at low and high temperatures with emphasis on the effect of $\mathrm{Re}$ concentration.

\section{Materials and Methods}

A series of Mo-Re alloys was prepared by powder metallurgy methods followed by hot and warm rolling. High-purity Mo and Re powders of $99.99 \mathrm{mas} \%$ were used to produce the green billets. The pressed billets were first annealed in flowing hydrogen. Then the purified billets were sintered in a vacuum and into flowing hydrogen once again. The sintered ingots were processed by a multistage rolling with intermediate heating to produce the sheet of $1 \mathrm{~mm}$ thickness. The technology used provides the following impurity concentrations: $\mathrm{Si}, \mathrm{Cr}$ and $\mathrm{Ni}<5 \mathrm{ppm} ; \mathrm{Fe}$ and $\mathrm{C} \sim 10$ ppm; $\mathrm{N}<1 \mathrm{ppm}$, and $\mathrm{O} \sim 10 \mathrm{ppm}$. A total of five materials with Re concentrations varied from $15.9 \mathrm{wt} \%$ to $50.1 \mathrm{wt} \%$ were produced and studied. In accordance with nominal Re concentrations, the prepared alloys were marked as: Mo-16Re, Mo-21Re, Mo-32Re, Mo-44Re and Mo-50Re.

Electron beam welding was conducted by the melt-run process (also known as bead on plate method) to simulate the practical welding using an electron beam welding apparatus. The welding parameters were as follows: accelerating voltage of $70 \mathrm{kV}$, beam current of $40 \mathrm{~mA}$, and beam 
movement rate of $2 \mathrm{~m} / \mathrm{min}$. The tensile specimens of $25 \mathrm{~mm}$ in length, with the gauge part $7.6 \times 1.5 \times 1 \mathrm{~mm}$, were used in welding experiments. One pass weld penetration process, from the top to the bottom up to a half depth of the specimen, was performed in the middle of the gauge part in a crosswise direction. The widths of weld-fusion zone and heat-affected zone were $0.7-1.0 \mathrm{~mm}$ and $0.4-0.5 \mathrm{~mm}$ respectively.

After welding, welded specimens were annealed for $1 \mathrm{~h}$ at two different temperatures, namely $1173 \mathrm{~K}(1473 \mathrm{~K}$ for Mo-50Re alloy) and $1673 \mathrm{~K}$ (1873 K for Mo-50Re alloy). The heat treatments used provide either stress-relieved or recrystallized structure for the base metal of welds.

Annealed welds were exposed to neutron irradiation. The irradiation at low (393-433 K) and high (1023-1073 K) temperatures up to fluencies of $3.6-7.3 \times 10^{25} \mathrm{n} / \mathrm{m}^{2}(\mathrm{E}>0.1$ $\mathrm{MeV}$ ) was carried out in the high-flux research reactor SM at the Research Institute of Atomic Reactors (RIAR, Russia). Such exposures produce damage levels $<1$ displacement per atom (dpa).

Unirradiated and irradiated at low and high temperatures specimens were tested by tensile tests at room temperature and nearby the irradiation temperatures in vacuum $\sim 0.1 \mathrm{~Pa}$ at a strain rate of $0.016 \mathrm{~s}^{-1}$. The microhardness of individual weld zones, namely weld metal (WM), heat-affected zone (HAZ) and base metal (BM), was measured before and after irradiation. In addition metallographic and TEM investigations of all three representative zones were performed.

\section{Results}

Measured values for ultimate tensile strength (UTS) and total elongation have been collected for unirradiated and irradiated alloys in Fig. 1 and Fig. 2 respectively. The UTS of unirradiated alloys decreases with testing temperature. Rhenium-rich alloys usually show higher levels of UTS in comparison with low-Re materials for all testing temperatures due to the so-called "Re effect". At the same time, the elongation of Re-rich specimens is higher at room temperature, while low-Re specimens exhibit better plasticity at elevated temperatures.

High-temperature (HT) irradiation causes hardening in all materials tested at room temperature (Fig. 1). The UTS of HT-irradiated specimens increases by $20-30 \%$ compared to the UTS of unirradiated counterparts. At the same time, low-temperature (LT) irradiation produces catastrophic embrittlement of all alloys. Half of radiation-exposed specimens lost their integrity during LT-irradiation. Few available specimens showed abnormally low strength. The strength of unirradiated alloys usually reduces with temperature. In contrast to unirradiated specimens, irradiated materials have a tendency to strengthen with temperature. For example, the UTS of HT-irradiated alloys increases by 1.2-2 times with elevation of the test temperature from $293 \mathrm{~K}$ to $1023 \mathrm{~K}$. Even after LT-irradiation, the strength increases with temperature at least in high-Re materials. For example,
Re-rich alloys are completely embrittled at room temperature. However, their strengths at $673 \mathrm{~K}$ show comparable results with those of unirradiated alloys.

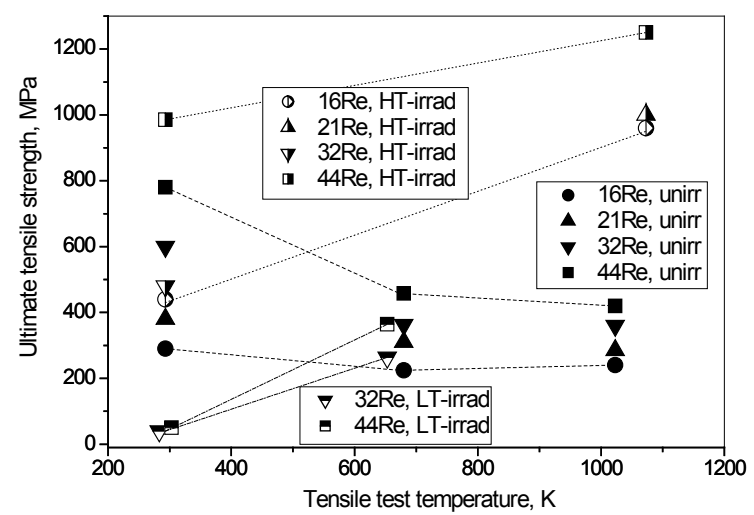

Figure 1. UTS for unirradiated (unirr) and irradiated at low (LT-irrad) and high (HT-irrad) temperatures Mo-Re alloys as a function of tensile test temperature

The elongations of both unirradiated and HT-irradiated specimens increase with testing temperature, demonstrating similar behaviour (Fig. 2).

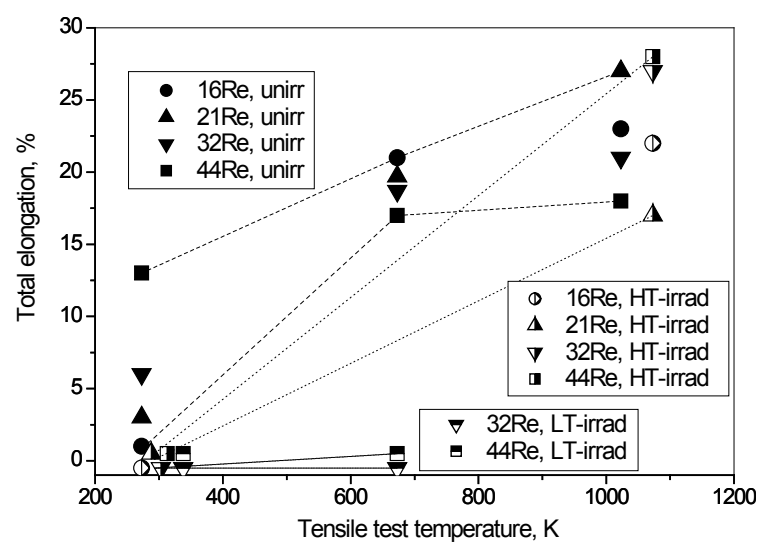

Figure 2. Total elongation for unirradiated (unirr) and irradiated at low (LT-irrad) and high (HT-irrad) temperatures Mo-Re alloys as a function of tensile test temperature

At room temperature, all unirradiated specimens have an advantage over irradiated ones. Their elongation increases with Re from $1 \%$ in Mo-16Re to $13 \%$ in Mo-44Re, while elongation of all HT-irradiated alloys does not exceed $1 \%$. However, plasticity of HT-irradiated alloys improves more actively with temperature. As a result, being tested close to the irradiation temperature, Re-rich irradiated specimens show higher elongations than their unirradiated counterparts. As mentioned before, LT-irradiated samples were absolutely brittle at room temperature. They also showed no signs of macroplasticity at $673 \mathrm{~K}$. Therefore, an increase in the test temperature to $673 \mathrm{~K}$ was insufficient to improve the ductility of alloys after LT-irradiation.

The microhardness of WM, HAZ and BM was measured at room temperature to estimate the influence of irradiation on the properties of various weld zones (Fig. 3). 

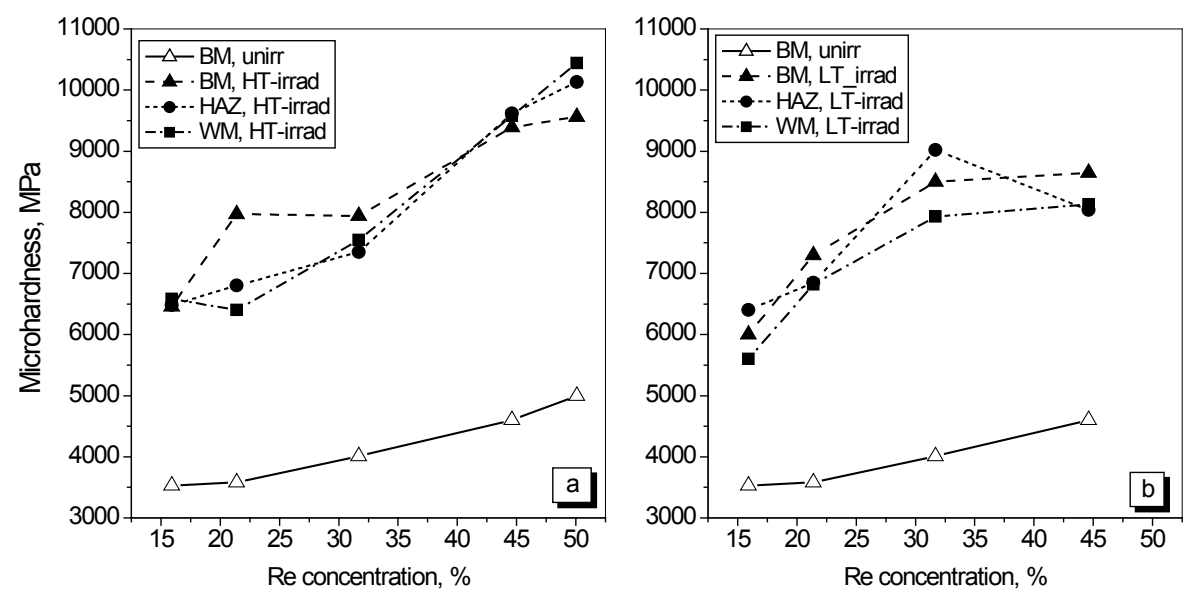

Figure 3. Microhardness of various welding zones for HT-irradiated (a) and LT-irradiated (b) Mo-Re alloys as a function of Re concentration

A few basic conclusions follow from the results obtained. First, the microhardness of unirradiated alloys monotonously increases with $\mathrm{Re}$ content. The difference in hardness between low-Re and high-Re alloys does not exceed $30 \%$. Second, irradiation at both low and high temperatures produces similar effects on the hardness of unirradiated alloys, though the hardness of HT-irradiated specimens is usually a bit higher than that of LT ones. Third, all weld zones in LT-irradiated and HT-irradiated alloys show very similar levels of microhardness for materials of the same compositions: fluctuations do not usually exceed $5-8 \%$, a figure which is within the observation accuracy range.

The irradiation essentially increases the difference in hardness between low-Re and high-Re alloys. Whereas such a difference is within $30 \%$ for unirradiated materials, it rises to $50 \%$ after both LT-irradiation and HT-irradiation. Rhenium alloying monotonously increases the hardness of LT-irradiated specimens with some saturation above $32 \%$ Re. The hardness of alloys irradiated at HT usually increases with the amount of Re for the whole concentration range.

The irradiation temperature influences the actual fracture mode as follows from the results of fractographic examination of tensile specimens after room temperature tests [16]. All specimens irradiated at high temperatures demonstrate transgranular fracture irrespective of the alloy composition. More signs of the microplasticity appear on the fracture surface with an increase of Re.

Low-temperature irradiation causes brittle intergranular fracture in Mo-16Re, a mixed mode in alloys with $21-32 \%$ $\mathrm{Re}$, and mainly transgranular fracture in Mo-44Re.

\section{Discussion}

\subsection{Hardening Mechanisms in Irradiated Mo-Re Alloys}

As follows from the results of mechanical tests, radiation-induced hardening (RIH) takes place in irradiated Mo-Re alloys. The effect of RIH consists of a drastic increase in the yield strength. This phenomenon has been already observed in a number of studies [9-13]. The RIH is strongly pronounced after HT-irradiation while it is feebly marked in LT-irradiated samples. A certain margin of ductility is necessary in practice to realise the RIH mechanism. If the ductility is very low, the flow stress is elevated above the inherent fracture stress of the material so that brittle fracture is observed before the true yield stress can be reached. Therefore, the RIH is very difficult to observe in alloys tested below the ductile-to-brittle transition temperature (DBTT).

The hardening of Mo-Re alloys is realised by three main mechanisms.

1) Solid solution hardening ( $\mathrm{SSH})$ is caused by interactions between solute atoms and dislocations. These interactions can be classified in parelastic interactions resulting from the interaction between the dilatation of the lattice near the solute atom and the dilatation around the stress field of a dislocation; dielastic interactions which are the consequences of local changes of bonding energies between the solute atom and the matrix; interactions resulting from atomic ordering; chemical effects and interactions resulting in changes of the local electronic structure. Without going into problems of radiation-induced generation of microstructural defects, all the above-listed interactions are operative in both unirradiated and irradiated alloys.

At room temperature Mo-Re alloys show a solid solution softening at Re concentrations up to $3 a t \%[7,17]$. At higher concentrations, rhenium alloying produces a moderate $\mathrm{SSH}$ of the matrix in the studied temperature range $300-800 \mathrm{~K}$ [17]. Since rhenium solubility in the Mo matrix is rather extended, the effect of SSH contributes significantly to the strength of alloyed Mo-Re materials increasing monotonously from a minimum in a vicinity of 3 at $\%$ throughout the whole single phase concentration range. Under irradiation conditions, the effect of SSH is operative as long as the solid solution remains stable. However, the mechanism of SSH cannot singly provide the observed intensity of RIH.

2) Hardening via the Hall-Petch mechanism is based on 
the interaction of moving dislocations with grain boundaries and subgrains. As a result, the yield strength in polycrystals increases with decreasing grain size. In addition to strength, structure refinement usually improves ductility, fracture toughness, corrosion resistance and fatigue. For example, in Mo-base alloys, which are known to be susceptible to brittle intergranular fracture, a larger grain size and coarser precipitates result in a higher DBTT, while finer inclusion particles result in a lower DBTT.

Evidently, the observed RIH cannot be explained by activation of the Hall-Petch hardening in the course of irradiation because no essential refinement of grains results from the neutron exposure. In contrast to the standard environment, a dispersed structure may even weaken hardening in irradiated fine crystalline alloys. Finer grains provide closer spacing of grain boundaries that are neutral sinks for the point defects produced by the irradiation. This could lower the number density and size of voids and loops, and reduce the effectiveness of these defects as hardening barriers. This conclusion is supported by the literature data. For example, irradiated high-purity Mo with a fine grained, elongated grain structure was shown to result in a low post-irradiated DBTT $[10,18]$, and, vice-versa, Mo single crystals demonstrate stronger hardening effect in comparison with polycrystals of similar compositions [19].

However, the Hall-Petch hardening has a strong influence on the properties of alloys with a heterogeneous granular structure. Fracture of Mo or Mo-Re welds is a good, and practically important, illustration of this problem. As is known, a grain size in different weld zones may range from a few tens of microns that are typical for the base metal, through tens to a hundred microns in the HAZ, to a sub-millimetre scale in the WM of electron-beam welds (Fig. 4). For example, the average grain sizes in Mo-21Re alloy were as follows: $20 \mu \mathrm{m}$ in the WM, $40 \mu \mathrm{m}$ in the HAZ and $350 \mu \mathrm{m}$ in the WM. In as-welded wrought Mo-alloys deformation is limited to the WM and HAZ [5]. This is due to the difference in strength between weld zones and inhomogeneous deformation caused mainly by heterogeneity in grain sizes.

Pre-weld and especially post-weld heat treatments at temperatures close to the recrystallization temperature are the effective means to recover the mechanical properties of Mo and Mo-Re welds [5-7]. The optimal post-weld annealing relieves localised strains and eliminates structural heterogeneity. The base metal becomes homogeneously recrystallized. The weld metal and the HAZ are both annealed and rearranged during the post-weld heat treatment. The edge of the HAZ seems to be epitaxial and continuous to the base metal. As a result, the boundary between the BM and HAZ is blurred, thus equalizing the strengths of both zones.

Grain growth limits the influence of the Hall-Petch strengthening in a range of high temperatures. Rhenium is known to elevate the recrystallization temperature. Grain refinement during directional solidification after welding is clearly observed with an increase in the Re content (Fig. 4).
Therefore, the potential influence of the Hall-Petch mechanism on the hardening of irradiated Mo-Re alloys is more pronounced in Re-rich alloys than in low-Re materials.

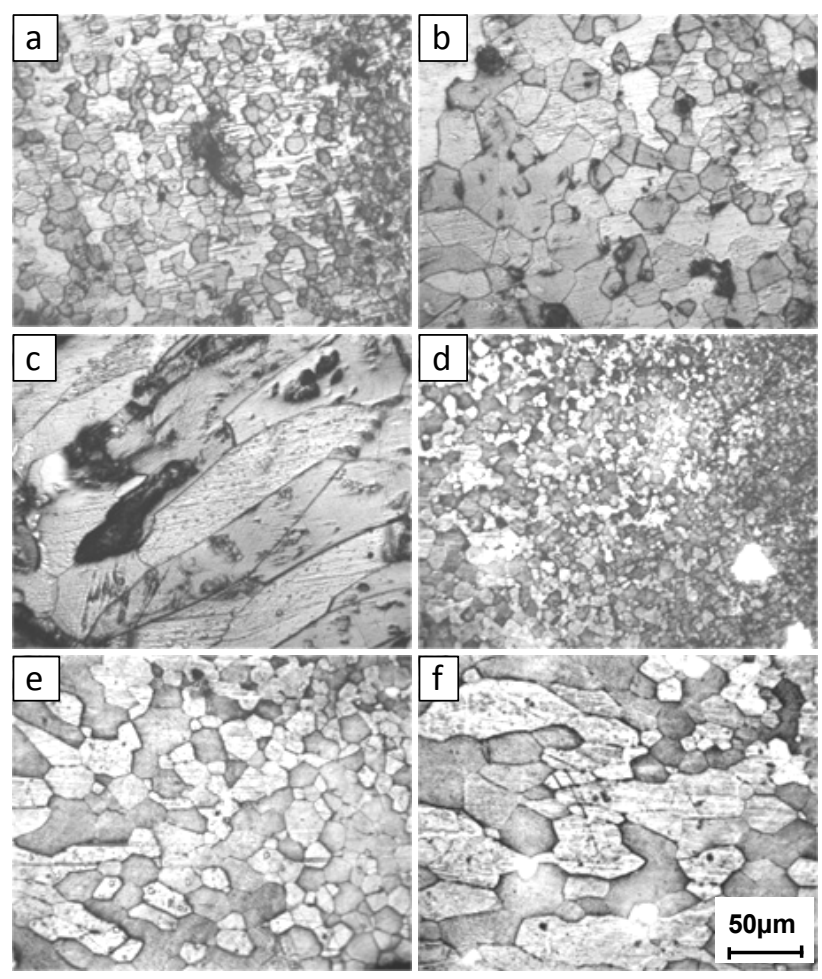

Figure 4. Optical micrographs of Mo-Re welds after post-weld annealing at $1173 \mathrm{~K}, 1 \mathrm{~h}$ and neutron irradiation at 1023-1073 K. Mo-32Re: a) BM, b) HAZ, c) WM and Mo-50Re: d) BM, e) HAZ, f) WM

3) The hardening of the matrix by the Orowan mechanism is connected to the interaction of moving dislocations with dispersive particles located within the grains along the dislocation path. The effectiveness of the Orowan mechanism at elevated temperatures is limited by the rate of coarsening or dissolution of strengthening phases and thus depends on their physical and chemical properties. Under irradiation conditions, the Orowan strengthening is still operative but its effect is dependent on the radiation-induced evolution of the microstructure and hence is temperature-dependent.

Dislocation loops and voids are formed during neutron irradiation of Mo-based alloys at relatively low temperatures, such as $400-800 \mathrm{~K}[8,11,20]$. The lower the irradiation temperature, the higher the number density, as well as the smaller the defect size. The fine voids and loops formed in a vicinity of $600 \mathrm{~K}$ are the most effective barriers to dislocation motion. They produce the strongest hardening and elevation of DBTT. In the case of single crystalline Mo-Re, an increase in the yield stress can be seen at a dose level as low as $5 \times 10^{-4}$ dpa even though the defect clusters responsible are too small to be resolved in the TEM [9]. The high number density of fine dislocation loops and voids may prevent the matrix from plastically deforming. Once cracks nucleate on grain boundaries and then brittle fracture occurs by transgranular cleavage across grains. 
In Mo-Re alloys, the effect of dislocation loops and voids depends on the Re concentration (Fig. 5a,b). With increasing rhenium content from $16 \%$ to $41 \%$ in LT-irradiated Mo-Re alloys, the density of dislocation loops with an average size of $7.5 \sim 10 \mathrm{~nm}$ is reduced by $4 \sim 6$ times from $4.5 \times 10^{21}$ to $2.6 \times 10^{22} \mathrm{~m}^{-3}[14]$. The space distribution of dislocation loops is extremely irregular in the $\mathrm{BM}$ of Mo-41Re weld, while these defects are not practically observed in the WM zone.

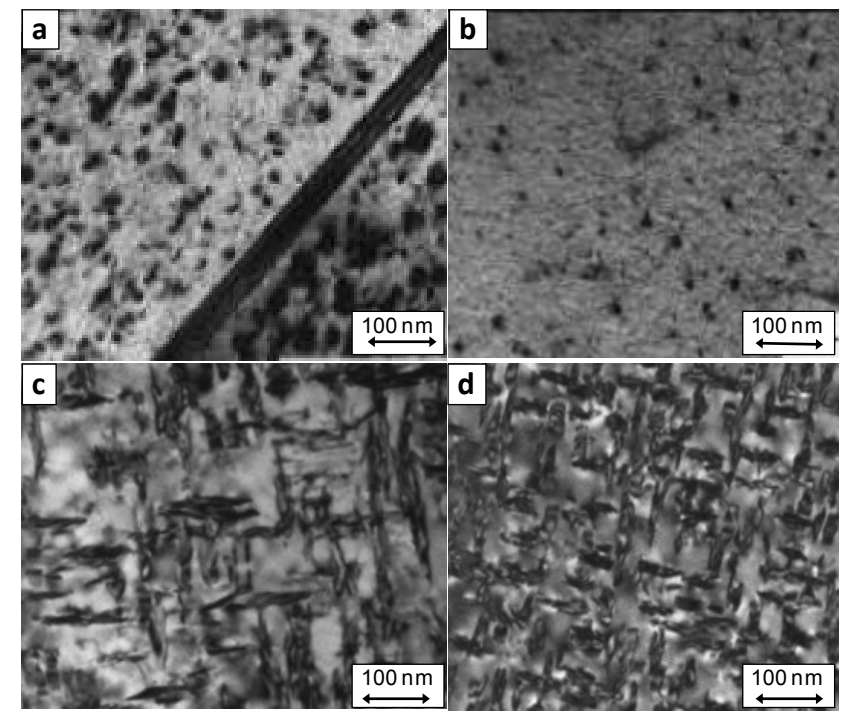

Figure 5. TEM microstructures (bright field) of dislocation loops in LT irradiated Mo-15Re (a) and Mo-41Re (b) and second phase particles in HT-irradiated Mo-15Re (c) and Mo-41Re (d) welds

The hardening from the voids can be calculated using the classical Orowan equation [21]. However, the calculated and experimental results show a good correlation only on the assumption that a certain part of the voids acts as hard Orowan obstacles while the rest are cut by moving dislocations. Evidently, the voids are not rigid and indestructible particles. Moreover, the question arises as to whether dislocation sources are able to generate fresh dislocations in sufficient number for plastic deformation. The microstructures of as-irradiated specimens before and after tensile tests were compared for TZM and Mo-Re alloys in TEM experiments to detect any effect of the tensile test on the microstructure [8]. Clear signs of deformation were found in as-tested TZM samples. On the contrary, no evidence of dislocation generation and motion was observed in as-tested Mo-5Re. The density and distribution of fine radiation-induced loops were identical to those in the unstrained counterpart. This suggests that the ability of the matrix to deform plastically is strongly restricted by the difficulty in generating mobile dislocations.

\subsection{Effect of Irradiation Temperature}

Self-interstitial atom (SIA) clusters readily nucleate on the periphery of collision cascades in a variety of irradiated bcc metals [22-24]. They subsequently perform fast thermally-activated one-dimensional motion. The one-dimensional glide of SIA clusters causes decoration of dislocations by SIA clusters/loops. At a rather low dose around $10^{-3} \mathrm{dpa}$, a large portion of the initially glissile clusters is trapped around slip dislocation loops. Nearly all of them become immobile. The high concentration of SIA loops around slip dislocations results in an extremely inhomogeneous spatial distribution of these defects [9]. The SIA decoration reduces the efficiency of dislocation sources to generate mobile dislocations. This leads to localization of plastic deformation giving rise to plastic instability, reduction in ductility and brittle fracture.

One may suppose that the mechanism of SIA decoration is evinced in Mo-Re alloys irradiated at lower temperatures. The Mo-Re matrix is hardened by small radiation-induced defects as can be seen from the microhardness measurements (Fig. 3). However, the tensile tests show no plasticity because all of the dislocation sources are unable to generate mobile dislocations (Fig. 2). The crack nucleation is not determined by plastic deformation and can only occur with flaws in the material. Once nucleated, the crack will propagate very rapidly through the material meeting with no resistance because no dislocations are generated at the crack tip.

Therefore, the LT neutron irradiation induces a loss of ductility and fracture is likely to depend on the presence of internal flaws. The material fails well below the yield strength demonstrating a very low strength level (Fig. 1). The measured values of tensile stress are actually a fracture stress which is a function of flaw size and the inherent toughness of the material. Spread in size of pre-existing flaws results in spread in values of the fracture stress. Within the data scatter, the fracture stress is independent of test temperature as long as the test is performed below the DBTT. In case of welds, more subcritical cracks, such as micropores or other flaws, are accumulated in coarse grains of WM compared to BM or HAZ. Therefore, the fracture surface will preferably be located within the weld metal, as is observed in the experiment [14].

Small radiation-induced loops and voids coarsen and their density rapidly decreases with an increase of the irradiation temperature above $1073 \mathrm{~K}$ in low-alloyed Mo [21,26]. Higher irradiation doses also coarsen dispersive defects. Evidently, coarsened voids produce a rather limited influence on the tensile properties and hence the hardening effect can diminish with an increase of the irradiation temperature. The mobility of point defects also increases with temperature. Therefore, thermally activated annihilation or recovery of properties occurs at a faster rate at higher temperatures.

Both coarsening and annihilation essentially reduce the hardening effect at elevated temperatures. As a result, no hardening was observed in a number of Mo-based alloys irradiated above $973 \mathrm{~K}$, while all these alloys harden in the ordinary way below $973 \mathrm{~K}$ [25]. A hardness increment after irradiation at $673 \mathrm{~K}$ is approximately twice that which occurs after irradiation at $1073 \mathrm{~K}$ for polycrystalline molybdenum and approximately half as much again for single crystals [19]. 
Evidently, no mechanisms, compensating degradation of the fine point defect clusters, are operative in low-alloyed Mo at elevated temperatures.

In contrast to low-alloyed materials, Mo-Re alloys exhibit a pronounced hardening after irradiation at both low and high temperatures $[14,15]$. This is seen from the results of microhardness measurement (Fig. 3). In contrast to LT-irradiation, Mo-Re alloys after HT-irradiation exhibit sufficient residual plasticity to amount to the yield strength in tensile tests. The strength of irradiated specimens being measured above the DBTT demonstrates a three- or four-fold increase compared to that of unirradiated materials (Fig. 1). The tensile tests at room temperature, which seems to be rather close to the DBTT, also demonstrate pronounced hardening of the matrix.

\subsection{Role of Radiation-Induced Decomposition of Mo-Re Solid Solution}

A new additional hardening mechanism takes effect in Mo-Re alloys during HT-irradiation to replace in part the diminished influence of coarsened point defect clusters. Most probably, such a mechanism is connected to intensive decomposition of Mo-Re solid solution. As was reported in many works $[11-13,20]$, precipitation of Re-rich phases after HT-exposure is observed for a wide range of $\mathrm{Re}$ concentrations in as-irradiated Mo-Re alloys, while all these alloys are single-phase materials in as-received states. The number of precipitates, their size and space distribution are a rather weak function of the Re content (Fig. $5 \mathrm{c}, \mathrm{d}$ ).

They all look like thin plates with a length $70-150 \mathrm{~nm}$ and are located in certain crystallographic planes. The particles are uniformly distributed over the bulk and no depleted zones are observed along the grain boundaries. Therefore, no preferred precipitation at structure defects or attachment to a particular weld zone is observed after HT-irradiation. Identification of these precipitates was not carried out in the present research. However, based on the previous works of irradiation assisted precipitation in Mo-Re alloys [12,20], the observed phases may be sigma phase (MoRe).

The phase transformations are initiated and promoted by radiation-induced segregation (RIS). The RIS effect on a free surface was studied experimentally in [27,28]. Rhenium atoms are transported toward the external surface during irradiation by an interstitial mechanism. Concurrently Re atoms are transported away from the surface by the vacancy flux due to the Kirkendall effect. Therefore, the net flux of Re atoms towards the external surface can only occur, if the transport of Re atoms toward the external surface by the interstitials is faster than the outward diffusion by vacancies. This condition restricts the temperature range of existence of RIS within $1073-1773 \mathrm{~K}$ for Mo-7Re or $1072-1500 \mathrm{~K}$ for Mo-30Re [27].

On the one hand, the RIS causes fluctuations in Re-concentrations in volumes which are sufficient for homogeneous nucleation. On the other hand, a large number of point defect clusters distributed over the bulk are sinks for segregating atoms and they can act as centres for heterogeneous nucleation. Since less matter and a shorter time are necessary to form a heterogeneous nucleus, the heterogeneous decomposition has an advantage over the homogeneous one. However, the availability of sinks is not a necessary condition for the phase transformation because very intensive RIS provides all the necessary conditions for homogeneous decomposition. After nucleation of fine precipitates, their surface acts as a sink for under size elements, such as Re atoms, leading to the growth of the precipitates.

Intensive decomposition of Mo-Re solid solution allows one to explain the observed equalization of properties of all zones of welds. As is known, the weldment usually fails through the weld metal because this zone is usually characterised by the lowest level of strength. As is seen from Fig. 3, HT-irradiation equalizes the hardness of all zones due to simultaneous decomposition of solid solution throughout the whole volume of weldment. As a result, the fracture crack may randomly propagate through any zone of as-irradiated weld without preferences.

\section{Conclusion}

The low-temperature neutron irradiation leads to a hard embrittlement of Mo-Re alloys. Tensile tests at 293-673 K exhibit no signs of macroscopic plasticity for all alloy compositions. The fracture mode changes from brittle intergranular fracture in low-Re alloys through a mixed mode to predominantly brittle transgranular fracture in high-Re materials.

At high-temperature neutron irradiation, the tensile strength of both unirradiated and irradiated Mo-Re welds typically increases with Re content. Almost all welds fail in a brittle transgranular manner at room temperature and demonstrate $20-30 \%$ elongation after high-temperature tensile tests. The HT-irradiation induces strong additional hardening of Mo-Re welds compared to unirradiated materials. The effect of radiation-induced hardening is evident at high-temperature tensile tests and is practically independent of the Re content. Limited ductility of welds suppresses the hardening effect at room temperature.

The microhardness of irradiated and unirradiated welds usually increases with the Re content. All weld zones of irradiated specimens show a severe radiation-induced hardening compared to that of unirradiated welds. The value of hardening is virtually identical for all weld zones and is practically independent of the irradiation temperature. The results of microhardness measurements clearly demonstrate that both LT and HT-irradiations harden the matrix of Mo-Re alloys. The lack of ductility impedes realisation of the hardening in tensile tests below the DBTT.

The radiation-induced hardening and loss of ductility after LT-irradiation are caused by a combined effect of the formation of irradiation-induced point defect clusters and the lack of mobile dislocations due to blocking of dislocation 
sources with decorated defects. The material fails well below the yield strength. The measured values of UTS are very low and they depend on the characteristics of available internal flaws.

Radiation-induced hardening at HT-irradiation is mainly connected to the intensive precipitation of Re-rich phases and their homogeneous distribution throughout the bulk. The precipitation is initiated and promoted by radiation-induced segregation of $\mathrm{Re}$ atoms. The damaging effect of HT-irradiation is similar in magnitude for all zones of Mo-Re welds.

\section{Acknowledgements}

The authors would like to express their gratitude to Dr V.P. Chakin for carrying out radiation experiments with the use of the SM reactor at RIAR, Russia. One of the authors (FM) would appreciate his gratitude to Frantsevich-IPMS for the Visiting Scientist during these studies.

\section{REFERENCES}

[1] R.I. Jaffee, C.T. Sims, J.J. Harwood. The effect of rhenium on the fabricability and ductility of molybdenum and tungsten, Proc. 3rd Int. Plansee Seminar, Benesovsky F (ed.), Plansee Metal AG, Reutte/Tirol, 380-411, 1958.

[2] W.D. Klopp. Review of ductilizing of group VIA elements by rhenium and other solutes, NASA Technical Note-4955. Lewis Research Centre, Cleveland, Ohio, December 1968. 32 p.

[3] A.A.F. Tavassoli. Present limits and improvements of structural materials for fusion reactors a review, Journal of Nuclear Materials, V.302, 73-88, 2002.

[4] M.S. El-Genk, J.M. Tournier. A review of refractory metal alloys and mechanically alloyed-oxide dispersion strengthened steels for space nuclear power systems, Journal of Nuclear Materials, V.340, 93-112, 2005.

[5] F. Morito. Characteristics of EB weldable molybdenum and Mo-Re alloys, JOM, V.45, 54-58, 1993.

[6] F. Morito. Weldability and fracture of Mo-50\%Re welds, Proc. 14th Int. Plansee Seminar, Kneringer G, Rödhammer P, Wilhartitz P (eds.), Reutte, V.1, 1037-1049, 1997.

[7] F. Morito F. Structures and properties of molybdenum alloys, Rhenium and Rhenium Alloys, Bryskin B (ed.), The Minerals, Metals \& Materials Society, 559-568, 1997.

[8] B.N. Singh J.H. Evans, A. Horsewell, P. Toft, D.J. Edwards. Microstructure and mechanical behaviour of TZM and Mo-5\%Re alloys irradiated with fission neutrons, Journal of Nuclear Materials, V.223, 95-102, 1995.

[9] B.N. Singh J.H. Evans, A. Horsewell, P. Toft, G.V. Mueller. Effects of neutron irradiation on microstructure and deformation behaviour of mono- and polycrystalline molybdenum and its alloys, Journal of Nuclear Materials,
V.258-263, 865-872, 1998.

[10] A. Hasegawa, K. Abe, M. Satou, K. Ueda, C. Namba. Influence of heat-treatment on tensile behavior of neutron irradiated molybdenum, Journal of Nuclear Materials, V.233-237, 565-569, 1996.

[11] Y. Nemoto, A. Hasegawa, M. Satou, K. Abe, Y. Hiraoka. Microstructural development and radiation hardening of neutron irradiated Mo-Re alloys, Journal of Nuclear Materials, V.324, 62-70, 2004.

[12] Hasegawa, K. Abe, M. Satou, C. Namba. Tensile behavior and microstructure of neutron-irradiated Mo-5\% Re alloy, Journal of Nuclear Materials, V.225, 259-266, 1995.

[13] D.J. Edwards, F.A. Garner, D.S. Gelles. The influence of neutron irradiation in FFTF on the microstructural and microchemical development of $\mathrm{Mo}-41 \mathrm{Re}$ at $470-730^{\circ} \mathrm{C}$, Journal of Nuclear Materials, V.375, 370-381, 2008.

[14] V.P. Chakin, F. Morito, V.A. Kazakov, Yu.D. Goncharenko, Z.E. Ostrovsky. Radiation embrittlement of Mo-Re welds under low-temperature irradiation in SM reactor, Journal of Nuclear Materials, V.258-263, 883-888, 1998.

[15] F. Morito, V.P. Chakin, M.I. Danylenko, A.V. Krajnikov. Radiation-induced strengthening in EB welds of Mo-Re alloys during high temperature neutron irradiation, Journal of Nuclear Materials, V.417, 976-979, 2011.

[16] A.V. Krajnikov, F. Morito, M.I. Danylenko. Embrittlement of molybdenum-rhenium welds under low and high temperature neutron irradiation, Journal of Nuclear Materials, V.444, 404-415, 2014.

[17] I. Wesemann, A. Hoffmann, T. Mrotzek, U. Martin. Investigation of solid solution hardening in molybdenum alloys. International Journal of Refractory Metals and Hard Materials, V.28, 709-715, 2010.

[18] B.V. Cockeram, R.W. Smith, K.J. Leonard, T.S. Byun, L.L. Snead. Irradiation hardening in unalloyed and ODS molybdenum during low dose neutron irradiation at $300^{\circ} \mathrm{C}$ and $600^{\circ} \mathrm{C}$, Journal of Nuclear Materials, V.382, 1-23, 2008.

[19] K. Watanabe, A. Hishinuma, Y. Hiraoka, T. Fujii. Neutron irradiation embrittlement of polycrystalline and single crystalline molybdenum, Journal of Nuclear Materials, V.258-263, 848-852, 1998.

[20] A. Hasegawa, K. Ueda, M. Satou, K. Abe. Neutron irradiation embrittlement of molybdenum rhenium alloys and their improvement by heat treatment, Journal of Nuclear Materials, V.258-263, 902-906, 1998.

[21] B.V. Cockeram, R.W. Smith, N. Hashimoto, L.L. Snead. The swelling, microstructure, and hardening of wrought LCAC, TZM, and ODS molybdenum following neutron irradiation, Journal of Nuclear Materials, V.418, 121-136, 2011.

[22] M. Wen, N.M. Ghoniem, B.N. Singh. Dislocation Decoration and Raft Formation in Irradiated Materials Philosophical Magazine (A), V.85, 2561-2580, 2005.

[23] B.N. Singh, A.J.E. Foreman, H. Trinkaus. Radiation hardening revisited: role of intracascade clustering, Journal of Nuclear Materials, V.249, 103-115, 1997.

[24] Yu.N. Osetsky, D.J. Bacon, B.N. Singh, B. Wirth. Atomistic 
study of the generation, interaction, accumulation and annihilation of cascade-induced defect clusters, Journal of Nuclear Materials, V.307-311, 852-861, 2002.

[25] T.S. Byun, M. Li, B.V. Cockeram, L.L. Snead. Deformation and fracture properties in neutron irradiated pure Mo and $\mathrm{Mo}$ alloys, Journal of Nuclear Materials, V.376, 240-246, 2008.

[26] B.V. Cockeram, R.W. Smith, L.L. Snead. The influence of fast neutron irradiation and irradiation temperature on the tensile properties of wrought LCAC and TZM molybdenum, Journal of Nuclear Materials, V.346, 145-164, 2005.

[27] R.A. Erck, L.E. Rehn. Kinetics of radiation-induced segregation in molybdenum-rhenium alloys, Journal of Nuclear Materials, V.168, 208-219, 1989.

[28] R.A. Erck, L.E. Rehn. Chi-phase precipitation in ion-irradiated molybdenum-rhenium alloys, Philosophical Magazine (A), V.62, 29-51, 1990. 